The Canadian Journal of Higher Education La revue canadienne d'enseignement supérieur

Volume XXXII, No. 3, 2002 pages $49-84$

\title{
Types et trajectoires d'insertion socioprofessionnelle de jeunes diplômés: Caractéristiques et profil sociodémographique*
}

\section{GENEVIEVE FOURNIER, ${ }^{\dagger}$ RENÉ PELLETIER,${ }^{\dagger}$ \& CHANTALE BEAUCHER ${ }^{\triangle}$}

tUniversité Laval, U Université du Québec à Montréal

\section{RÉSUMÉ}

Cet article aborde les résultats d'une étude menée auprès de jeunes diplômés des secteurs secondaire professionnel, collégial technique et universitaire, $1^{\text {er }}$ cycle, en processus d'insertion socioprofessionnelle. Il vise à rendre compte des différents événements personnels et professionnels qui ponctuent l'entrée sur le marché du travail de ces jeunes diplômés et à faire état de leur situation professionnelle trois ans après leur sortie du système éducatif. Plus particulièrement, nous présentons d'abord quatre types et onze trajectoires d'insertion socioprofessionnelle identifiés à partir d'entretiens semi-structurés, effectués trois ans après l'obtention du diplôme. Nous proposons ensuite une analyse de ces résultats en fonction du sexe et du niveau de scolarité des sujets. Finalement, une discussion concernant les formes plus ou moins insidieuses que prend la précarité pour une proportion importante de diplômés et une réflexion sur les groupes qui y sont les plus vulnérables complètent l'article.

* Cette recherche a été réalisée grâce à une subvention octroyée par le Conseil de Recherches en Sciences Humaines du Canada (CRSH). 


\section{ABSTRACT}

This article discusses the results of a study conducted toward young graduates from professional high school, technical college and university during the first three years of their socio-professional integration process. The purpose is to understand different events, personal and professional, that are significant when young graduates enter to the work market. Moreover, their professional situation three years after graduation is discussed. More closely, we present four types and eleven paths of socioprofessional integration, identified from interviews carried out three years after graduation. By means of these interviews, we propose an analysis of the results according to the gender and level of education of the subjects. Finally, a discussion about the insidious form of professional instability for a growing proportion of graduates, as well as a reflection about the different groups that are more vulnerable to it, is presented.

Au cours des dernières décennies, la place du travail et son rôle normatif dans les sociétés occidentales industrialisées ont été remis en question (Chalifour, 1997; Fournier \& Bourassa, 2000). Ainsi, malgré la reprise de la croissance économique de la fin des années 1990 , caractérisée par la hausse du taux d'emploi et la baisse du taux de chômage (Fortier, 2000), la structure économique qui s'est installée au début des années 1980 semble avoir irrémédiablement bouleversé les modalités d'insertion des nouveaux travailleurs. En effet, il est maintenant généralement admis que ce qui a d'abord été perçu comme une crise conjoncturelle se révèle plutôt être de nature structurelle, une véritable mutation (Aubry, 1998; Castel, 1995; Conseil permanent de la jeunesse, 2001; Gorz, 1997; Khan, 1997; Onimus, 1997). Par ailleurs, si tous sont touchés à des degrés divers, cette réorganisation affecte plus durement encore les jeunes adultes qui tentent de faire leur place sur le marché de l'emploi (Bovin, 1995; Perret, 1995), même les détenteurs d'un diplôme (Fournier, Monette, Pelletier \& Tardif, 2000).

Ainsi, en 1999, 46,5\% des emplois atypiques étaient occupés par des jeunes de 15 à 29 ans (Conseil permanent de la jeunesse, 2001). Or, la plupart de ces emplois se caractérisent par l'absence d'un lien d'emploi 
stable avec une organisation, d'une garantie d'embauche une fois le contrat terminé et d'une protection syndicale; ils sont aussi généralement de courte durée et mal rémunérés. Cette situation fait en sorte que de nombreux jeunes expérimentent la pauvreté; en fait, $36,3 \%$ des prestataires de l'assurance-emploi ont entre 15 et 29 ans (Conseil permanent de la jeunesse, 2001). Malheureusement, la récurrence des périodes de chômage au moment de l'insertion des jeunes peut les entraîner dans un cercle vicieux de plus en plus pénalisant:

La récurrence du chômage n'apporte pas d'atouts particuliers pour l'insertion future. Elle stigmatise, en revanche, l'individu par exclusion du marché primaire, celui des emplois plus stables et mieux rémunérés. Les chômeurs récurrents sont, dès lors, surtout confrontés au marché secondaire avec des épisodes de chômage plus brefs succédant à des périodes d'emplois elles aussi plus brèves. Le marché du travail, tel un filtre, intégrerait progressivement les individus selon une employabilité décroissante. (Lollivier, 2000, p. 49).

Pourtant, la quête de stabilité semble demeurer l'objectif de la majorité des nouveaux travailleurs. Parmi les stratégies envisagées pour faire face à l'incertitude du marché de l'emploi, de nombreux jeunes optent pour un allongement de leur scolarité ou un retour à l'école après un certain temps au travail (Nicole-Drancourt, 1997). Toutefois, bien que de nombreux auteurs soulignent le lien positif entre l'augmentation de la scolarisation et l'accès à un emploi (Béret, 1996; Bovin, 1995; Pair, 1998; Rebière, 1998; Trottier, Perron \& Diambomba, 1995), il semble que plusieurs jeunes subissent malgré tout les effets du déclassement des diplômes, engendré notamment par le déséquilibre entre l'offre et la demande d'emploi (Baby, 2000; Pair, 1998; Rebière, 1998). En outre, afin de faire face aux impératifs financiers de la vie, plusieurs d'entre eux sont obligés d'accepter des emplois sans débouché, sans lien avec leur formation initiale et ne leur apportant ni stabilité, ni satisfaction personnelle (Tremblay, 1994). Le diplôme ne constitue donc plus un passeport pour l'emploi stable, pas plus qu'il ne garantit une insertion socioprofessionnelle satisfaisante qui fait écho aux attentes des diplômés. 
Dans ce contexte, l'insertion socioprofessionnelle de plusieurs diplômés s'accompagne de phases d'instabilité marquées par une flexibilité forcée, qui s'est intensifiée en même temps que le marasme économique s'enracinait. Dès lors, bon nombre d'entre eux expérimentent une période d'incertitude de plus en plus longue au cours de laquelle ils ne sont ni totalement insérés, ni totalement exclus, mais plutôt dans une zone intermédiaire, quelque part entre les deux. Comme le rapportent Fournier et Bourassa (2000), ils se situent dans un espace creux, une sorte de flou ou de non lieu identitaire, difficile à définir, à cerner et à catégoriser. Dans la même foulée, Rose (2000) fait remarquer qu'il existe actuellement un déplacement notable des frontières entre emploi, chômage, formation et inactivité et que:

souvent, les statuts (salarié, scolaire, chômeur) ne restituent pas convenablement la réalité des situations (des chômeurs travaillent, des scolaires ont des petits boulots, des salariés sont en formation... (p. 111 ).

Qu'en est-il alors des nouveaux diplômés qui tentent de faire leur place sur le marché du travail? Quelles sont les distinctions et ressemblances significatives dans la pluralité de leurs trajectoires d'insertion socioprofessionnelle et dans la manière de les vivre? Les hommes et les femmes ont-ils des trajectoires semblables? Telles sont quelques-unes des questions auxquelles cherche à répondre cet article. Plus spécifiquement, nos objectifs sont de mettre en relief les différents types et trajectoires d'insertion socioprofessionnelle d'un groupe de jeunes diplômés ${ }^{1}$ et de rendre compte de certaines différences observées, selon le sexe et le niveau de scolarité.

\section{Le processus d'insertion socioprofessionnelle: un champ de recherche en ébullition}

En dépit de l'intérêt des chercheurs pour cette problématique, l'entrée des jeunes dans la vie active demeure un champ de recherche relativement nouveau, qui suscite de nombreux débats et questionnements (Rose, 1998, 2000). L'instabilité des structures d'emploi, l'ampleur du chômage et la complexité des modes d'insertion, appellent en effet au renouvellement des conceptualisations et des 
interventions. Elles exigent que l'étude du processus d'insertion socioprofessionnelle des jeunes ne se limite plus uniquement à l'utilisation d'indicateurs comme l'adéquation formation/emploi ou la dichotomie actif/inactif pour rendre compte de la pluralité des situations vécues. De fait, la définition traditionnelle de l'insertion socioprofessionnelle, élaborée en période de plein emploi, tient difficilement la route si on prend en compte les transformations structurelles du marché du travail et leurs nombreux effets tant sur le nombre d'emplois disponibles que sur les caractéristiques objectives (statut, salaire, type de contrat, etc.) et subjectives liées à l'exercice de ces emplois (reconnaissance des compétences, occasions de réalisation personnelle, sentiment d'appartenance, etc.). Ainsi, Trottier (2000), faisant état des données récentes sur l'insertion socioprofessionnelle, résume ainsi ce processus:

Or, les analyses de l'insertion professionnelle comme processus ont montré que l'entrée dans la vie professionnelle ne s'effectue pas toujours de façon linéaire, et qu'elle est caractérisée par des discontinuités, par une diversité de modes de fréquentation scolaire et d'insertion professionnelle qui ne sont pas toujours conciliables avec une conception linéaire du cours de la vie. (p. 36)

Par ailleurs, la définition même du processus d'insertion crée difficilement consensus. En effet, les sources de confusions et de malentendus qui règnent dans ce domaine sont révélatrices d'une difficulté essentielle: le point de départ de ce processus est imprécis et son aboutissement n'est pas clair (Vincens, 1998). Ainsi, pour certains, il suppose que les individus ont trouvé un travail stable, en lien avec leur formation et correspondant à leur projet de vie (Vincens, 1998). Pour d'autres, ce processus s'achève lorsque l'individu a atteint une position stabilisée sur le marché du travail, c'est-à-dire qu'il occupe durablement des positions instables mais qui lui permettent d'acquérir une expérience professionnelle qualifiante (Vernières, 1993). Quelques-uns estiment que pour considérer l'individu vraiment inséré, il importe d'abord et avant tout que l'emploi occupé corresponde à la formation reçue (Laflamme, 2000). D'autres accordent moins d'importance à cette relation dans le processus 
d'insertion socioprofessionnelle, ou du moins lui reconnaissent une importance relative (Rose, 2000; Trottier, 2000). Enfin, quelques auteurs insistent sur l'importance de prendre en compte à la fois des variables objectives telles que le statut d'emploi ou son lien avec la formation et des variables plus subjectives, telles que la satisfaction ressentie vis-à-vis la situation professionnelle ou le sens accordé au travail, pour rendre compte de la fin de ce processus (Fournier, Jeanrie \& Croteau, 1999).

Enfin, si le processus d'insertion socioprofessionnelle demeure un objet d'études à développer, quelques éléments particulièrement significatifs émergent néanmoins de l'ensemble des études effectuées dans les dernières années: l'insertion socioprofessionnelle est une condition nécessaire à l'atteinte de l'autonomie financière et affective tout comme elle est une voie quasi incontournable d'accès à la citoyenneté (Fournier \& Bourassa, 2000; Paugam, 2000). Elle est progressive, incertaine, relativement diversifiée (Rose, 1998) et elle a un caractère aléatoire (Dagenais, 1998). Elle est tout, sauf un événement ponctuel (Fournier, Boivin \& Viel, 1999; Nicole-Drancourt, 1997; Rose, 1994). Par conséquent, la stabilisation professionnelle est retardée pour plusieurs (Nicole-Drancourt \& Roulleau-Berger, 2000) à un point tel que certains travailleurs risquent de devenir des «insérés à vie» (Fusulier, 1996). En ce sens, Nicole-Drancourt (1992) note l'éclatement et la restructuration en profondeur des anciens modèles d'insertion en différents itinéraires où dominent les dimensions d'incertitude, de flexibilité et de réversibilité. Certains auteurs parlent alors de précarisme pour traiter de ces parcours d'insertion qui maintiennent, de manière plus ou moins sévère, les jeunes dans des conditions globales de précarité ou qui, à l'inverse, en facilitent l'affranchissement (Canals, 1998).

\section{Quelques typologies de trajectoires d'insertion socioprofessionnelle}

L'importance d'une analyse des trajectoires d'insertion socioprofessionnelle est par ailleurs confirmée par la nécessité de repérer les états, leur permanence relative et leurs enchaînements. Aussi, bien qu'une certaine prudence soit de mise à cet égard, les auteurs soulignent le lien étroit entre les conditions d'entrée sur le marché du travail et la poursuite de l'ensemble de la vie active (Pottier, 1992; Rose, 1998). Plus 
particulièrement, pour Pottier (1992), les premiers événements qui interviennent à la sortie de l'école s'articulent les uns aux autres et, s'ils ne sont pas déterminés au départ, se répartissent rapidement entre deux trajectoires: une suite d'emplois qui conduisent progressivement à la stabilisation professionnelle et une forme d'alternance prolongée entre emplois et chômage qui mène, à des degrés divers, à une plus grande précarité professionnelle. Dans la même veine, Canals (1998) rapporte que le passage par des formes d'emplois atypiques chez les jeunes donne lieu au développement de deux types de trajectoires d'insertion socioprofessionnelle. D'une part, les trajectoires ascendantes où le contrat à durée déterminée et l'intérim constituent des formes contractuelles dans lesquelles s'effectuent les premières années de la vie active avant de déboucher sur l'intégration professionnelle durable. Pour Paugam (2000), ce type de trajectoires serait un moyen de parvenir à une intégration assurée et ne conduirait pas à la précarisation professionnelle. D'autre part, les trajectoires linéaires ou décroissantes, caractérisées par une récurrence entre des emplois à statut instable, des périodes de chômage et d'inactivités, qui conduiraient ou maintiendraient l'individu dans un état de précarité professionnelle plus ou moins prononcée.

D'autres auteurs se sont penchés sur l'expérience des jeunes entre le moment où ils quittent le système éducatif et celui où ils accèdent à un emploi satisfaisant. D'abord, Nicole-Drancourt et Roulleau-Berger (1995) ont dégagé trois familles de trajectoires d'insertion socioprofessionnelle chez les jeunes modestement diplômés au cours des deux premières années passées sur le marché du travail. Deux de ces trajectoires se situent aux opposés d'un continuum: d'un côté, les jeunes qui s'insèrent rapidement et qui conservent leur emploi longtemps $(28 \%$ des garçons et $35 \%$ des filles) et de l'autre côté, les jeunes exclus de l'emploi ( $11 \%$ des garçons et 19\% des filles). Au centre, on retrouve une forte proportion de jeunes en attente plus ou moins favorable, dont la trajectoire est instable et incertaine quant à leur devenir (NicoleDrancourt \& Roulleau-Berger, 1995).

De son côté, Gauthier (1994) remarque le peu de cas fait des situations multiples qui composent le processus d'insertion 
socioprofessionnelle des jeunes. Afin de combler cette lacune, l'auteure propose une typologie composée de sept trajectoires qui tiennent compte de la situation objective et de la représentation subjective que s'en font les répondants. La filière traditionnelle traduit la situation des individus qui ont eu accès assez rapidement à un emploi typique relativement stable. Ensuite, le parcours chaotique réfère aux individus qui ont vécu plusieurs expériences d'emplois, dans leur domaine d'études, en attendant un poste d'emploi plus typique et qui leur conviendrait davantage. Le troisième type de trajectoire, l'instabilité créatrice, fait référence aux jeunes ayant «utilisé» et contourné l'incertitude pour se bâtir une carrière, parfois leur entreprise. La stabilité dans la précarité constitue un quatrième type de trajectoire d'insertion socioprofessionnelle. Les individus qui s'y retrouvent ont choisi de faire «n'importe quoi» plutôt que de prendre le risque de ne «rien faire». Cette catégorie inclut les jeunes mères ou les étudiants qui choisissent de conserver un emploi à temps partiel afin de pouvoir investir leur temps et leur énergie ailleurs. Le cinquième type recensé par Gauthier (1994) est la fuite en avant. Il caractérise la situation des individus qui se sont lancés en affaires dans des secteurs innovateurs par crainte de la précarité et parce qu'ils ont cru que les possibilités d'accès à des emplois plus traditionnels étaient inexistantes. Mal armés pour s'attaquer à ce type d'emplois, ils se sont retrouvés en situation d'échec. La précarité comme état, le sixième type de trajectoire d'insertion socioprofessionnelle, fait référence à une situation d'emploi et de chômage intermittente et d'emplois atypiques mal rémunérés. Enfin, le dernier type, les exclus des statistiques, regroupe ceux qui sont «officiellement» sans emploi, mais qui travaillent «au noir», et les inactifs, dont les itinérants.

Ces quelques typologies de trajectoires d'insertion socioprofessionnelle ouvrent la porte à une meilleure compréhension de cette période de vie charnière. Elles témoignent de l'importance de saisir les nombreuses situations intermédiaires qui jalonnent les premières années de vie active, c'est-à-dire celles comprises entre l'exclusion et le travail stable, et de tenir compte, dans l'analyse de ces trajectoires, des significations qu'elles prennent pour les jeunes eux-mêmes. C'est dans 
cette perspective qu'a été élaborée la présente recherche. Nous avons voulu mettre en relief les premières années d'expérience en emploi de jeunes diplômés et rendre compte des événements professionnels et personnels qui jalonnent cette étape de vie. Nous avons cherché à cerner leur situation d'insertion trois ans après la sortie du système éducatif et à reconstruire les trajectoires qui ont mené à cette situation. Nous avons accordé une attention particulière à l'utilisation de dimensions objectives et subjectives pour identifier, avec le plus d'acuité possible, les nombreuses situations intermédiaires comprises entre l'exclusion socioprofessionnelle et la stabilisation socioprofessionnelle.

\section{METHODOLOGIE}

\section{Les sujets}

Les résultats de recherche présentés ici sont tirés d'une étude longitudinale (1993-1999) dont l'objectif premier était d'identifier les trajectoires d'insertion socioprofessionnelle de diplômés, sur une période de cinq ans. Au départ, les volontaires retenus devaient être en dernière année, à temps plein, d'un programme d'études menant directement à l'exercice d'un métier ou d'une profession - secondaire professionnel, collégial technique, baccalauréat spécialisé. Le choix des programmes d'études s'est fait selon les disponibilités des milieux scolaires et des enseignants, en évitant toutefois le plus possible les secteurs d'études traditionnellement associés à un sexe particulier: coiffure, génie mécanique, etc. Les sujets ont été rejoints en classe et ils ont été invités à participer à l'étude, sur une base volontaire. En tout, 205 sujets provenant de 20 programmes d'études différents (sept programmes d'études secondaires, six d'études collégiales et sept d'études universitaires) ont constitué l'échantillon initial et ont participé à la première cueillette de données. Après 18 mois, les sujets ont été invités à compléter un questionnaire portant sur leur situation professionnelle (i.e., en emploi, chômage, statut de l'emploi, lien avec la formation, satisfaction, etc.). À la troisième année de l'étude, en plus de compléter à nouveau un questionnaire portant sur leur situation professionnelle, les sujets ont été rencontrés en entretien individuel. Enfin, à la cinquième 
année, les sujets ont complété le questionnaire relatif à leur situation professionnelle. Les résultats présentés ici sont tirés des entretiens semistructurés auxquels 151 personnes, sollicitées environ trois ans après la fin de leurs études, ont accepté de participer. ${ }^{2}$

L'échantillon est formé de $71 \%$ de femmes $(n=108)$ et de $29 \%$ d'hommes $(n=43)$. Parmi les 151 répondants, $37(24 \%)$ ont suivi une formation professionnelle au secondaire, 57 (38\%) ont suivi une formation technique au collégial alors que les 57 autres $(38 \%)$ ont complété une formation de premier cycle à l'université. Il est à souligner que 7 sujets ( $5 \%$ ) n'ont pas obtenu leur diplôme de fin d'études et qu'ils sont tous de niveau collégial.

\section{Cueillette des données}

Les données ont été récoltées par entretiens individuels, d'une durée moyenne de 90 minutes. Les questions ouvertes et semi-ouvertes visaient à établir une rétrospective des événements de la vie professionnelle et personnelle des sujets au cours de leurs trois premières années d'insertion sur le marché du travail. D'abord, les sujets ont rapporté, de manière séquentielle et factuelle, les différentes situations professionnelles vécues (ex. statut des emplois occupés, lien avec leur formation, périodes de chômage, de formation, d'inactivité, etc.). Ils se sont ensuite exprimés sur la façon dont ils ont vécu ces situations et les autres événements significatifs qui ont ponctué leur vie depuis leur entrée sur le marché du travail. Finalement, les sujets ont été invités à décrire leur situation professionnelle actuelle.

\section{Procédure d'analyse qualitative des données}

Une analyse préliminaire a permis d'acquérir une vue d'ensemble des données brutes, de se familiariser avec leurs particularités et de mettre en place un schéma de travail précis. Une fois les caractéristiques significatives circonscrites, l'analyse de contenu a été entreprise. Conformément aux procédures méthodologiques suggérées par L'Écuyer (1990) et aux éléments techniques proposés par Bardin (1991), cette démarche s'est déroulée en trois étapes. Dans un premier temps, nous avons procédé au codage afin d'organiser l'ensemble des données brutes 
obtenues au cours des entretiens avec les jeunes travailleurs selon la chronologie de leur trajectoire d'insertion socioprofessionnelle. Les données recueillies auprès des sujets ont toutes été retranscrites et ordonnées dans le cadre des situations d'emploi ayant jalonné leur insertion socioprofessionnelle. Dans un deuxième temps, la classification des informations ordonnées précédemment a permis de regrouper les trajectoires d'insertion socioprofessionnelle qui partagent les mêmes caractéristiques et ainsi, de discerner onze classes homogènes. À l'analyse subséquente (interprétation), des particularités et des dimensions particulières ont émergé plus clairement et ont permis la définition de quatre types d'insertion socioprofessionnelle et de onze trajectoires qui leur sont associées.

Par type d'insertion socioprofessionnelle, nous entendons la position qu'occupent les répondants après trois ans sur le marché du travail. Pour identifier et distinguer ces types d'insertion, deux dimensions plus objectives ont été retenues: (1) la stabilité du dernier emploi (par exemple: CDI ou CDD) $)^{3}$ et (2) le lien de cet emploi avec la formation choisie. Deux dimensions plus subjectives ont aussi été retenues: (1) la satisfaction plus générale ressentie dans le dernier emploi (en ce qui concerne les tâches; la reconnaissance des compétences, la valorisation au travail, etc.) et (2) la satisfaction financière (autonomie ou marge de manœuvre financière). L'ensemble de ces dimensions rend compte du type d'intégration en emploi des sujets trois ans après leur sortie du système scolaire.

Les trajectoires d'insertion socioprofessionnelle font référence, quant à elles, au cheminement effectué par les sujets entre la sortie du système éducatif et leur troisième année sur le marché du travail. Pour construire et distinguer ces trajectoires, cinq dimensions plus objectives ont été retenues: (1) le lien du premier emploi avec la formation; (2) la durée de la transition entre la fin de la formation et l'obtention d'un premier emploi; (3) le nombre d'emplois occupés et leur durée; (4) la durée des périodes d'inactivité ou de chômage et, (5) le lien des divers emplois occupés avec la formation. Six dimensions plus subjectives ont aussi été retenues: (1) les attentes vis-à-vis du travail; (2) la satisfaction vis-à-vis des emplois occupés; (3) le caractère choisi ou non des diverses 
situations professionnelles; (4) le degré de précarité professionnelle et financière ressenti; (5) la qualité perçue du réseau de soutien social et, (6) la qualité perçue du réseau de références socioprofessionnelles.

\section{RÉSULTATS}

La présentation des résultats est divisée en deux parties. Dans la première, nous décrivons les types et trajectoires d'insertion socioprofessionnelle des diplômés interrogés. Dans la deuxième partie, plus brève, la distribution des sujets selon leur sexe et leur niveau de scolarité est présentée.

\section{Types et trajectoires d'insertion socioprofessionnelle}

Afin de faciliter la compréhension des types et trajectoires d'insertion socioprofessionnelle identifiés, soulignons que chacun des quatre types d'insertion socioprofessionnelle regroupe deux ou trois trajectoires. Les caractéristiques générales du type d'insertion socioprofessionnelle sont décrites, puis les spécificités des trajectoires sont passées en revue.

L'insertion réussie $(\mathbf{4 0} \%)$. Après trois ans sur le marché du travail, les individus qui appartiennent à ce groupe occupent un emploi stable, pour la plupart dans leur domaine d'études. Ils possèdent des conditions de travail qu'ils considèrent avantageuses et se disent globalement très satisfaits de leur situation professionnelle. Leur situation financière, bien qu'ils souhaitent encore l'amélioration, est jugée satisfaisante et leur permet d'actualiser des projets dans leur vie personnelle. Ces individus se sentent valorisés par leur nouveau statut de travailleur et sont fortement investis dans les tâches et les responsabilités professionnelles qu'ils doivent remplir. Toutefois, plusieurs font face à de fortes pressions de performance et de rendement. Pour répondre à ces exigences, ils choisissent parfois de mettre entre parenthèses certains aspects de leur vie en dehors du travail et cherchent à se modeler aux exigences et aux attentes du milieu professionnel auquel ils appartiennent. Ce type d'insertion regroupe trois trajectoires particulières. 
La trajectoire progressive. Les sujets qui s'insèrent dans cette trajectoire connaissent une progression constante et graduelle vers la réalisation de leurs objectifs et de leur projet professionnel. Après un temps d'arrêt entre la vie scolaire et professionnelle variant de deux à six mois, ces personnes connaissent deux ou trois emplois successifs dans des entreprises différentes, mais tous liés à leur domaine d'études. D'un emploi à l'autre, les sujets obtiennent des conditions d'emploi de plus en plus satisfaisantes. Ils manifestent une très forte volonté de réalisation par le travail et se disent prêts à faire des sacrifices pour atteindre leurs objectifs. Au bout de trois ans, ces sujets ont le sentiment d'avoir acquis une expérience d'emploi valable dans leur domaine professionnel et perçoivent que celle-ci est reconnue par leur milieu. Ils ont développé un réseau de références professionnelles et personnelles solide. Finalement, ces sujets insistent sur leur qualité de vie et leur bien-être: la stabilisation professionnelle leur permet d'atteindre un équilibre entre les besoins liés au travail et ceux liés à la vie privée.

La trajectoire traditionnelle. Un cheminement linéaire et prévisible caractérise cette trajectoire d'insertion socioprofessionnelle. Dès la sortie de l'école, ces jeunes décrochent un emploi et connaissent dans les années suivantes deux ou trois emplois à durée indéterminée, liés à leur domaine de formation et toujours dans la même entreprise. Très rapidement, ils se sentent reconnus pour ce qu'ils font, autant par leurs collègues de travail que par leur employeur et ils ont le sentiment de participer à la réussite de l'entreprise, d'y avoir leur place. En retour, l'entreprise reconnaît leur valeur en leur offrant de la formation d'appoint, ce qui leur permet de gravir les échelons et d'améliorer leurs conditions d'emploi. En bout de ligne, les sujets ont le sentiment de pouvoir compter sur une stabilité professionnelle et économique leur permettant peu à peu d'investir certains projets personnels. Cependant, ils continuent à investir de façon prioritaire leur travail, parfois au détriment des autres sphères d'activités, pour répondre aux pressions de rendement et de performance de l'entreprise.

La trajectoire pragmatique. À leur entrée sur le marché du travail, ces sujets connaissent un temps de transition, caractérisé par l'incertitude et l'instabilité et qui s'échelonne sur deux ans. Au cours de cette période, 
ils obtiennent plusieurs emplois (plus de trois), plus ou moins liés à leur domaine d'études, précaires et entrecoupés par des périodes de chômage plus ou moins longues. Finalement, ne pouvant connaître de progression ni de stabilisation professionnelle, ils abandonnent leur projet professionnel initial. Par la suite, ils se positionnent dans un domaine professionnel différent du leur et, face aux nouvelles tâches et responsabilités à accomplir, ils se découvrent des intérêts insoupçonnés. Ils structurent alors un nouveau projet professionnel qui les satisfait et se trouvent alors stabilisés dans une entreprise où ils comptent faire carrière. Après trois ans sur le marché du travail, ces travailleurs se considèrent professionnellement et financièrement autonomes et sécurisés, de même qu'en pleine possession de leur vie. Ils ont par ailleurs un réseau social et un réseau de références professionnelles sur lesquels ils estiment pouvoir compter et qui les ont soutenus au cours des années plus chaotiques qu'ils ont connues.

L'insertion sinueuse (15\%). Durant les trois années suivant la fin de la formation, ces personnes ont connu des débuts plutôt difficiles bien qu'elles aient participé de manière relativement récurrente au marché du travail. Elles ont occupé de deux à plus de quatre emplois dont le lien avec la formation est très variable et le plus souvent, entrecoupés de courtes périodes de chômage. Après trois ans, ces sujets occupent un emploi qui, bien qu'instable et pas toujours lié à leur formation, est jugé satisfaisant. En effet, la situation de travail, malgré ses difficultés, est perçue comme convenable parce qu'elle a été choisie et qu'elle est relativement bien assumée. Du point de vue de ces sujets, les choix professionnels faits au cours de la troisième année constituent une réponse valable à leurs attentes, compte tenu du peu d'opportunités offertes sur le marché du travail. Toutefois, les sujets n'en prétendent pas moins à davantage de sécurité professionnelle et financière et à de meilleures conditions de travail. Avec plus ou moins de succès, ces jeunes travailleurs tentent de maintenir un équilibre entre les diverses exigences professionnelles et leurs investissements personnels. Enfin, ils bénéficient d'un soutien important de leur entourage et de leur famille. On compte deux trajectoires au sein de ce type d'insertion. 
La trajectoire relationnelle. Les sujets qui sont regroupés dans cette trajectoire remettent progressivement en question le sens et la place du travail dans leur vie et choisissent d'investir davantage leur vie privée. En effet, ils expérimentent à leur sortie de l'école une période sans emploi de deux à six mois, suivie de l'exercice de plusieurs emplois, liés de façon aléatoire au domaine d'études et plus ou moins précaires. Après trois ans, ils sont pour la plupart en emploi, lequel est souvent atypique et sans lien avec leur formation, mais généralement jugé satisfaisant parce qu'il n'entrave pas la mise en cuvre de projets personnels. Au travail, les tâches ont plus ou moins d'importance, pourvu qu'elles ne nuisent pas à leur qualité de vie et que l'emploi leur permette une entrée d'argent relativement régulière. En somme, les décisions prises dans la vie professionnelle reposent davantage sur le désir de parfaire ou de préserver la qualité des liens avec leur réseau social ou familial que sur la recherche d'un emploi stable, correspondant à leurs qualifications.

La trajectoire entrepreneuriale. Les sujets de la trajectoire entrepreneuriale font le choix de travailler à leur compte ou de créer leur propre entreprise au terme d'une période d'insertion houleuse où ils remettent en question leur statut de salarié. À leur arrivée sur le marché du travail, ces personnes vivent une période de transition qui varie entre trois et douze mois. Ils occupent ensuite un ou deux emplois jugés très insatisfaisants au terme desquels ils choisissent de démarrer une entreprise. Pour la plupart, ce choix est une réponse à la piètre qualité des emplois exercés et repose sur un désir de réalisation professionnelle, d'autonomie et d'un plus grand contrôle sur leur contexte de travail. Après trois ans sur le marché de l'emploi, les sujets expérimentent toujours une certaine inquiétude financière et une précarité professionnelle. Ils acceptent cependant relativement bien cette situation, ainsi que l'importante charge de travail qu'ils affrontent quotidiennement, parce qu'ils considèrent qu'elle est temporaire, normale et choisie. Ils se sentent en outre valorisés personnellement et professionnellement. Enfin, plusieurs répondants font état de la qualité du soutien moral et financier qu'ils reçoivent de leur entourage. 
L'insertion essoufflante (18\%). Après trois ans sur le marché du travail, ces sujets occupent un emploi stable sur le marché du travail. Cependant, cet emploi n'est pas en lien avec leur formation et il est jugé insatisfaisant ou très insatisfaisant, notamment en ce qui concerne les tâches à accomplir qui exigent un niveau de qualification plus bas que le leur. Ces tâches sont également jugées ingrates, ennuyantes voire dévalorisantes et même dangereuses. Cette situation est soit occasionnée par des événements personnels inattendus (séparation, difficultés à rembourser des dettes étudiantes, etc.) qui obligent les personnes à abandonner leurs objectifs et à travailler «à tout prix», soit par une série d'échecs et de déceptions dans la recherche d'un emploi satisfaisant, en lien avec la formation et surtout économiquement viable. En somme, ils sont contraints à l'abandon de leur projet professionnel pour obtenir une certaine stabilité financière. Si certains d'entre eux se trouvent relativement satisfaits de leur situation financière, tous espèrent que leur situation professionnelle s'améliorera à long terme et qu'ils pourront s'épanouir au travail. En fait, ils estiment que leur situation en est une d'attente et qu'elle s'est imposée pour des raisons hors de leur contrôle. On compte deux types de trajectoires au sein de ce type d'insertion.

La trajectoire économique. Les sujets qui sont regroupés dans cette trajectoire se caractérisent par leur besoin impératif d'atteindre une indépendance économique et de remplir leurs obligations financières, responsabilités qui pour eux, se situent en amont de la réalisation du projet d'insertion socioprofessionnelle. En effet, l'arrivée sur le marché du travail de ces jeunes est marquée par l'avènement d'obligations financières qui les obligent à mettre en attente leur projet de carrière et à rechercher un travail pouvant leur assurer une stabilité professionnelle et économique, peu importe le domaine. Après trois ans sur le marché du travail de façon récurrente (sans période de transition) et à la suite de compromis importants, tous les sujets occupent un emploi stable, mais ils considèrent celui-ci peu valorisant et peu satisfaisant. Néanmoins, ils ont le sentiment d'avoir réussi partiellement leur insertion: ils peuvent rencontrer leurs obligations financières. Enfin, ils considèrent que leur situation est temporaire et circonstancielle et qu'ils auront éventuellement accès à un emploi qui leur convient davantage. 
La trajectoire moratoire. Les sujets qui font partie de cette trajectoire sont en emploi de manière relativement récurrente: le moment de transition est relativement court ( $0-6$ mois), ils connaissent peu de périodes d'inactivité et elles sont plutôt brèves. Cependant, les emplois occupés sont mal rémunérés et très insatisfaisants, à tous les égards. Découragés, désemparés, et face à la nécessité de gagner leur vie, ils abandonnent leur projet professionnel pour accepter un emploi peu gratifiant, dans un autre domaine que le leur. Cependant, cet emploi est stable et leur assure une certaine autonomie financière. Tous les sujets qui s'insèrent dans la trajectoire moratoire se trouvent confrontés à la même impasse: ils se voient forcés d'accepter un emploi peu gratifiant pour ne pas précariser davantage leur situation économique déjà peu solide. En contrepartie, ils n'acquièrent pas d'expériences de travail valables dans leur domaine et s'éloignent de plus en plus d'une stabilisation professionnelle dans le secteur d'études choisi. En outre, ils se sentent isolés et mentionnent ne pas pouvoir compter sur un réseau de références professionnelles. Par ailleurs, ils développent des relations amicales et sociales intéressantes, parfois sur les lieux de travail.

L'insertion chaotique $(\mathbf{1 6 \%})$. N'arrivant pas à atteindre une certaine stabilité professionnelle et l'autonomie financière, les sujets de ce type d'insertion possèdent peu d'expériences de travail valables ou signifiantes après trois ans sur le marché du travail. Ceux qui travaillent occupent un emploi instable et généralement de très courte durée, dont les tâches sont jugées sans intérêt. Tous sont installés d'une manière assez sévère dans la précarité professionnelle et financière. Tout au long des trois années, ils ont expérimenté plusieurs périodes d'inactivité ou de chômage et ils ont exercé des emplois peu rémunérateurs et de courte durée. Pour ces sujets, l'avenir à court terme n'est jamais assuré et l'avenir à moyen ou long terme est inenvisageable: le parcours est hachuré, imprévisible et sans véritable fil conducteur. D'une part, il y a ceux qui font face à des embûches structurelles répétées et qui abandonnent leurs objectifs professionnels initiaux. D'autre part, il y a ceux qui manquent d'habiletés et de ressources nécessaires à l'atteinte de leurs objectifs professionnels dans un marché de l'emploi compétitif et contraignant. Les uns et les autres vont différer leurs besoins personnels, 
compte tenu de la fragilité et de la précarité économique qui caractérisent leur situation socioprofessionnelle. De façon distinctive, tous ces jeunes sont à la recherche d'un espace dans la sphère de la vie au travail et d'une position professionnelle plus stable, gratifiante et économiquement viable. Ce type d'insertion est constitué de trois trajectoires spécifiques.

La trajectoire précaire. Ces sujets ne connaissent pas de véritable progression sur le marché du travail et la transition entre la formation scolaire et la vie professionnelle peut durer jusqu'à dix-huit mois. Ensuite, ils effectuent des allers-retours entre des périodes d'emploi et de chômage plus ou moins longues ( 1 à 10 mois). Leur trajectoire, discontinue et incertaine, est marquée par l'occupation de plus de trois emplois instables, atypiques, peu rémunérés et décevants, mais la plupart du temps dans leur domaine professionnel. À ce propos, certains ont refusé de «bons emplois» parce qu'ils n'étaient pas liés à leur formation. Ces jeunes ont en effet la particularité de s'en tenir très étroitement à leur projet professionnel et de rechercher dans leur travail une occasion d'actualiser les compétences spécifiques acquises durant la formation. La succession, la recherche continuelle et le cumul d'emplois font en sorte que les sujets souffrent du stress qu'occasionnent les adaptations successives aux différents milieux de travail. Plusieurs peuvent par ailleurs compter sur le support de leur réseau social, mais leur réseau de références professionnelles demeure faible. Devant la précarité de leur situation, ces personnes sont contraintes de repousser la réalisation de leurs projets personnels et de faire des concessions importantes dans leurs différentes sphères de vie.

La trajectoire d'exclusion. La trajectoire d'exclusion regroupe les sujets qui connaissent durant leur parcours une suite d'embûches graves, la plupart du temps liées à des facteurs structurels du marché du travail, face auxquels ils se trouvent dépourvus, mais liées aussi parfois à des difficultés à s'ajuster à certaines règles du travail (respect des horaires, exécution des tâches, prise de responsabilités, observation des consignes, etc.). À leur sortie du système éducatif, ces sujets sont inquiets face à leur démarche d'insertion socioprofessionnelle et ils ont l'impression que leur seule possibilité d'action repose sur la chance et le hasard. Or, 
en ce qui les concerne, ils n'ont pas de chance. En outre, le moment de transition entre la diplômation et l'obtention du premier emploi est très long (6-18 mois). Il est suivi d'une succession de quelques emplois (deux ou trois) dans un autre domaine que le leur, de très courte durée et peu valorisants: «des emplois que personne ne veut ou que n'importe qui pourraient exercer, sans diplôme». Ces expériences ont pour conséquences de miner leur moral et de contribuer à affaiblir leur sentiment de compétence comme travailleur. Aucun sujet de cette trajectoire n'a de projet d'action au moment de l'enquête et tous dépendent économiquement de leur réseau social, de l'aide financière et des programmes d'employabilité gouvernementaux.

La trajectoire exploratoire. Les sujets de la trajectoire exploratoire ont en commun d'avoir quitté le monde scolaire sans diplôme. Ils ont espéré trouver sur le marché du travail plus de motivation et de valorisation personnelle. Le désir d'atteindre l'autonomie sociale par le travail et d'acquérir un statut d'adulte les anime de façon prioritaire. Ils tentent de découvrir leur potentiel professionnel par essais et erreurs, selon les possibilités qui se présentent à eux. Durant les trois années passées sur le marché du travail, ils vont occuper peu d'emplois (deux ou trois) sans lien ni continuité, mal payés, s'exerçant dans des conditions difficiles et entrecoupés de périodes de chômage assez longues. Les sujets peinent à conserver un emploi et à s'ajuster aux exigences du marché du travail. Ils n'ont pas de projet professionnel et manifestent relativement peu de préoccupations face à l'avenir. Cependant, le simple fait d'être rémunérés pour les tâches qu'ils accomplissent entraîne une certaine valorisation face à leur statut de travailleur, même si leur instabilité, leurs revenus et leurs conditions d'emplois ne les satisfont pas.

\section{Le cas particulier de l'itinéraire scolaire}

Quelques individus $(n=17)$ reprennent, trois ans après l'obtention d'un premier diplôme, le chemin de l'école. Ce parcours constitue ainsi un cas à part puisque les sujets qui s'y trouvent se situent à une étape différente de leur cheminement par rapport à celui de leurs confrères et consœurs en processus d'insertion socioprofessionnelle. Les personnes 
qui choisissent d'effectuer un retour à l'école ont en quelque sorte «échoué» à leur première tentative. Puisqu'il n'est pas possible d'évaluer leur situation d'emploi à ce point précis, elle ne peut plus être comparée à celle des sujets des autres types d'insertion. Cependant, la trajectoire qui a précédé la décision de retourner à l'école se compare facilement à celle des sujets qui s'inscrivent dans la trajectoire précaire. En fait, si nous les avions interrogés peu de temps auparavant, ils s'y seraient insérés étant donné la multiplication d'emplois précaires occupés, leur instabilité financière, leurs conditions d'emplois insatisfaisantes, etc. Ainsi, les sujets qui choisissent de retourner aux études connaissent à un moment ou à un autre de leur cheminement une remise en question de leur projet de carrière initial. Insatisfaits de leur domaine professionnel et des possibilités de réalisation qu'il peut offrir, les sujets initient une réflexion qui impose, à leurs yeux, un retour aux études. Une fois leur décision prise, ils se disent heureux de leur choix et éprouvent un grand bien-être malgré certains inconvénients qui y sont rattachés, notamment la perte de leur autonomie financière, le report de certains projets personnels et souvent la dépendance vis-à-vis de la famille d'origine ou vis-à-vis du conjoint ou de la conjointe. Cette réorientation est appuyée par un réseau social aidant; ils ont dès lors la satisfaction d'avoir devant eux une perspective d'avenir intéressante.

Le tableau 1 résume l'ensemble des types d'insertion et des trajectoires qui y correspondent. Ces quatre types d'insertion et ces onze trajectoires d'insertion socioprofessionnelle étant décrits, nous procédons maintenant à une analyse de la répartition des sujets, selon deux dimensions sociodémographiques: le sexe et le niveau de scolarité des répondants.

\section{Distribution des sujets selon le niveau de scolarité.}

À prime abord, on constate, tel que le démontrent les résultats présentés au tableau 2, l'importance relative de la représentation des sujets issus du secondaire dans certaines trajectoires. D'abord, 60\% $(\mathrm{n}=23)$ des individus diplômés du secondaire professionnel se retrouvent dans trois trajectoires seulement: traditionnelle $(24 \%)$, moratoire $(22 \%)$ et progressive $(14 \%)$. D'autre part, la répartition des 
Tableau 1

Types et trajectoires d'insertion socioprofessionnelle

\begin{tabular}{|c|c|c|c|}
\hline \multirow{2}{*}{$\begin{array}{l}\text { Types d'insertion } \\
\text { socioprofessionnelle }\end{array}$} & \multirow{2}{*}{$\begin{array}{l}\text { Trajectoires d'insertion } \\
\text { socioprofessionnelle }\end{array}$} & \multicolumn{2}{|c|}{ Sujets } \\
\hline & & $n=151$ & $\%$ \\
\hline \multirow[t]{4}{*}{1 - Réussie } & & 61 & 40 \\
\hline & - Progressive & 34 & 26 \\
\hline & - Traditionnelle & 20 & 15 \\
\hline & - Pragmatique & 7 & 5 \\
\hline \multirow[t]{3}{*}{2 - Sinueuse } & & 22 & 15 \\
\hline & - Relationnelle & 14 & 9 \\
\hline & - Entrepreneuriale & 8 & 5 \\
\hline \multirow[t]{3}{*}{ 3-Essoufflante } & & 27 & 18 \\
\hline & - Économique & 7 & 5 \\
\hline & - Moratoire & 20 & 15 \\
\hline \multirow[t]{5}{*}{ 4-Chaotique } & & 24 & 16 \\
\hline & - Précaire & 16 & 12 \\
\hline & - Exclusion & 5 & 3 \\
\hline & - Exploratoire & 3 & 2 \\
\hline & - Scolaire & 17 & 11 \\
\hline
\end{tabular}

individus issus de programmes du secondaire laisse voir une polarisation dans les trajectoires «avantageuses», mais également, bien que dans une moindre mesure, dans des trajectoires beaucoup moins favorisées: moratoire $(22 \%)$, précaire ( $8 \%$ ) et d'exclusion ( $8 \%$ ). Ainsi, dans le cas particulier des personnes présentant une trajectoire traditionnelle ou progressive, qui se situent dans le type d'insertion réussie, la situation professionnelle est relativement stable et avantageuse. Pour la trajectoire moratoire, l'emploi est relativement stable, mais jugé insatisfaisant et peu gratifiant; pour les trajectoires précaire et exploratoire, la participation effective au marché du travail est mise en péril. 
Ensuite, plus des deux tiers des individus ayant fréquenté le cégep se retrouvent principalement dans quatre trajectoires d'insertion: scolaire ( $21 \%)$, progressive $(19 \%)$, moratoire $(16 \%)$ et précaire $(12 \%)$. De plus, on remarque que tous les sujets de la trajectoire exploratoire $(5 \%)$ ont suivi une formation collégiale et que ces trois hommes ont abandonné leur programme de formation avant son terme. On remarque également qu'un nombre important de sujets du collégial se trouvent dans une situation marginale après trois ans sur le marché du travail: soit parce qu'ils n'arrivent pas à se dénicher un emploi satisfaisant et qu'ils retournent à l'école ( $21 \%)$, soit qu'ils doivent se contenter d'emplois de survie $(28 \%)$.

Enfin, trois ans après avoir obtenu un diplôme de premier cycle à l'université, $75 \%$ de ces jeunes travailleurs se trouvent dans quatre trajectoires: progressive (32\%), traditionnelle (16\%), relationnelle $(16 \%)$ et précaire $(11 \%)$. En cumulant les trois premières trajectoires, on peut dire que $64 \%$ des universitaires se trouvent, trois ans après leur diplômation, dans une situation professionnelle qu'ils jugent plus qu'acceptable. Ceux qui appartiennent aux trajectoires progressive et traditionnelle sont stabilisés sur le marché de l'emploi où ils connaissent une progression satisfaisante et des conditions de travail avantageuses. Quant à ceux qui s'insèrent dans la filière relationnelle, bien que leur position professionnelle soit instable et précaire à certains égards, ils se disent globalement satisfaits de leur situation parce qu'elle est choisie et parce que les relations humaines, la vie de couple ou de famille ont davantage pris d'importance pour eux que la vie professionnelle. Enfin, il convient de noter qu'aucune des personnes de ce niveau scolaire ne se retrouve dans les trajectoires d'exploration et d'exclusion.

Enfin, en comparant les trajectoires des sujets des trois niveaux de scolarité, une ressemblance entre la situation des diplômés du secondaire et celle des diplômés universitaires, ressort d'évidence. En effet, $46 \%$ des individus du secondaire se trouvent dans le type d'insertion réussie, alors que $52 \%$ des universitaires occupent la même catégorie. Ils sont donc nombreux à être stabilisés sur le marché du travail et relativement à l'abri de la précarité professionnelle. Cependant, la situation des collégiens est moins favorable. D'abord, ils ne sont que le quart (26\%) à s'inscrire dans l'insertion réussie, ensuite, 


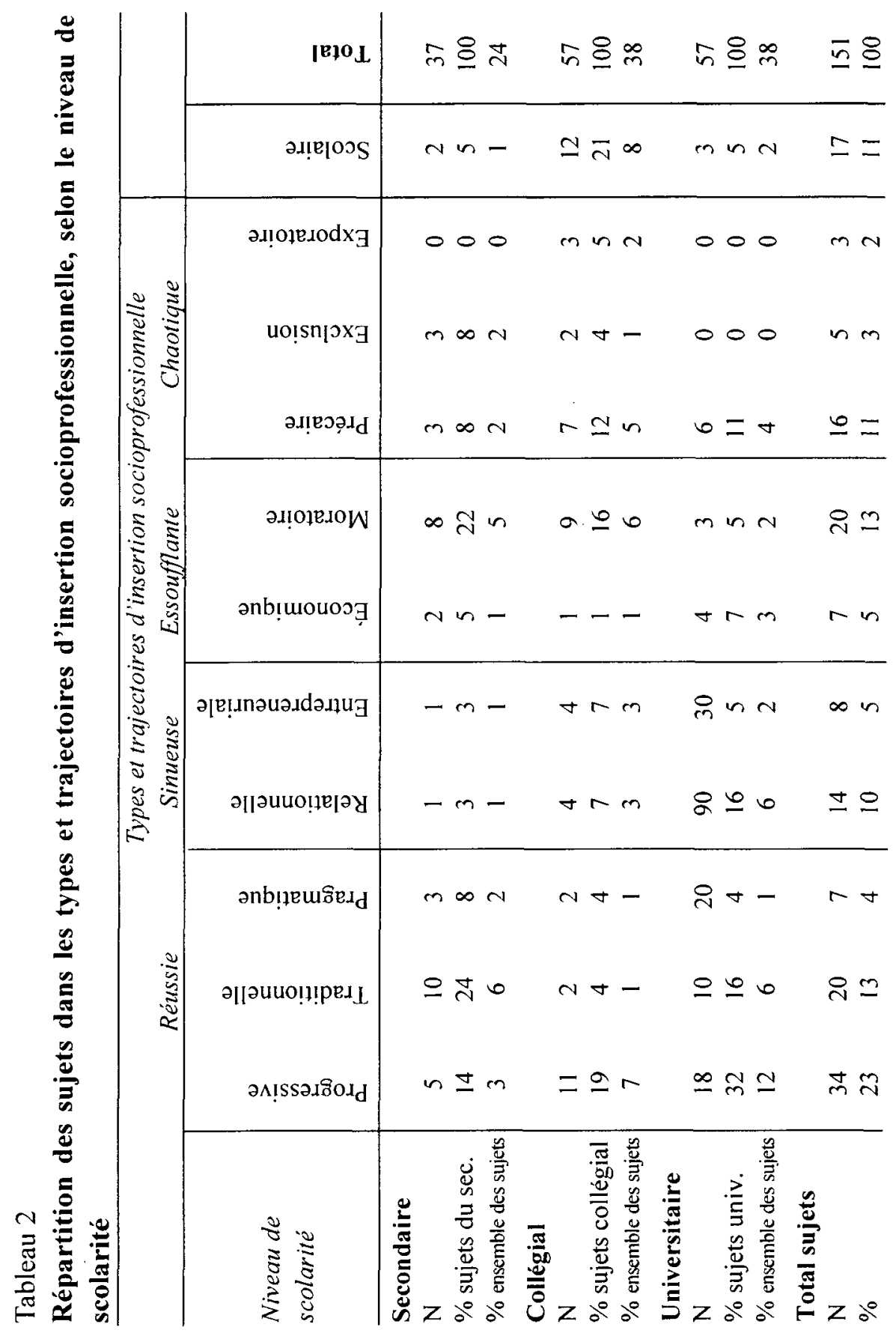

The Canadian Journal of Higher Education Volume XXXII, No. 3, 2002 
ils sont les plus nombreux à avoir expérimenté des trajectoires marquées par la précarité. Ainsi, 37\% de ceux-ci s'inscrivent dans les trajectoires moratoire $(16 \%)$, précaire $(12 \%)$, d'exclusion $(4 \%)$ et exploratoire (5\%). En outre, ils sont les plus nombreux (21\%) à avoir effectué un retour aux études après plusieurs tentatives infructueuses et décevantes d'insertion sur le marché de l'emploi.

\section{Distribution des sujets selon le sexe}

Les 151 sujets de cette étude se répartissent inégalement en fonction de leur sexe. Bien qu'une attention particulière à ce propos ait été portée dans le choix des programmes de formation au début de l'étude, il est apparu qu'une majorité de femmes ont accepté de participer (initialement $60 \%$ de femmes et $40 \%$ d'hommes). De plus, un nombre plus important d'hommes s'est progressivement retiré de l'étude au cours des années. Ainsi, l'échantillon de la troisième année de la recherche se compose de 108 femmes (72\%) et 43 hommes (28\%). Bien qu'il soit délicat de tirer des conclusions hâtives étant donné la différence de représentation des sexes dans cette étude, les données présentées au tableau 3 montrent un certain déséquilibre dans deux trajectoires d'insertion socioprofessionnelle. Dans un premier cas, il est remarquable de constater que les femmes accaparent complètement la trajectoire relationnelle. Aucun de leur confrère ne s'y trouve. Par contre, ces derniers sont significativement plus nombreux que les femmes à se situer dans la trajectoire économique. La fonction utilitaire du travail constitue un point de jonction entre ces deux trajectoires.

En outre, on ne peut passer sous silence une situation semblable dans la trajectoire exploratoire. En effet, seuls des hommes, non diplômés de surcroit (7\%), s'inscrivent dans cette trajectoire. Par ailleurs, on constate que les hommes sont plus polarisés autour des types d'insertion réussie (44\%) et essoufflante ( $23 \%$ ). Pour leur part, exception faite de l'insertion réussie où elles se retrouvent à $38 \%$, les femmes sont réparties de façon plus égale dans les types d'insertion sinueuse (18\%), essoufflante (16\%) et chaotique (16\%). Cependant, elles sont presque le double des hommes à se situer dans la trajectoire précaire $(\mathrm{F}=13 \% ; \mathrm{H}=5 \%)$. Enfin, la 


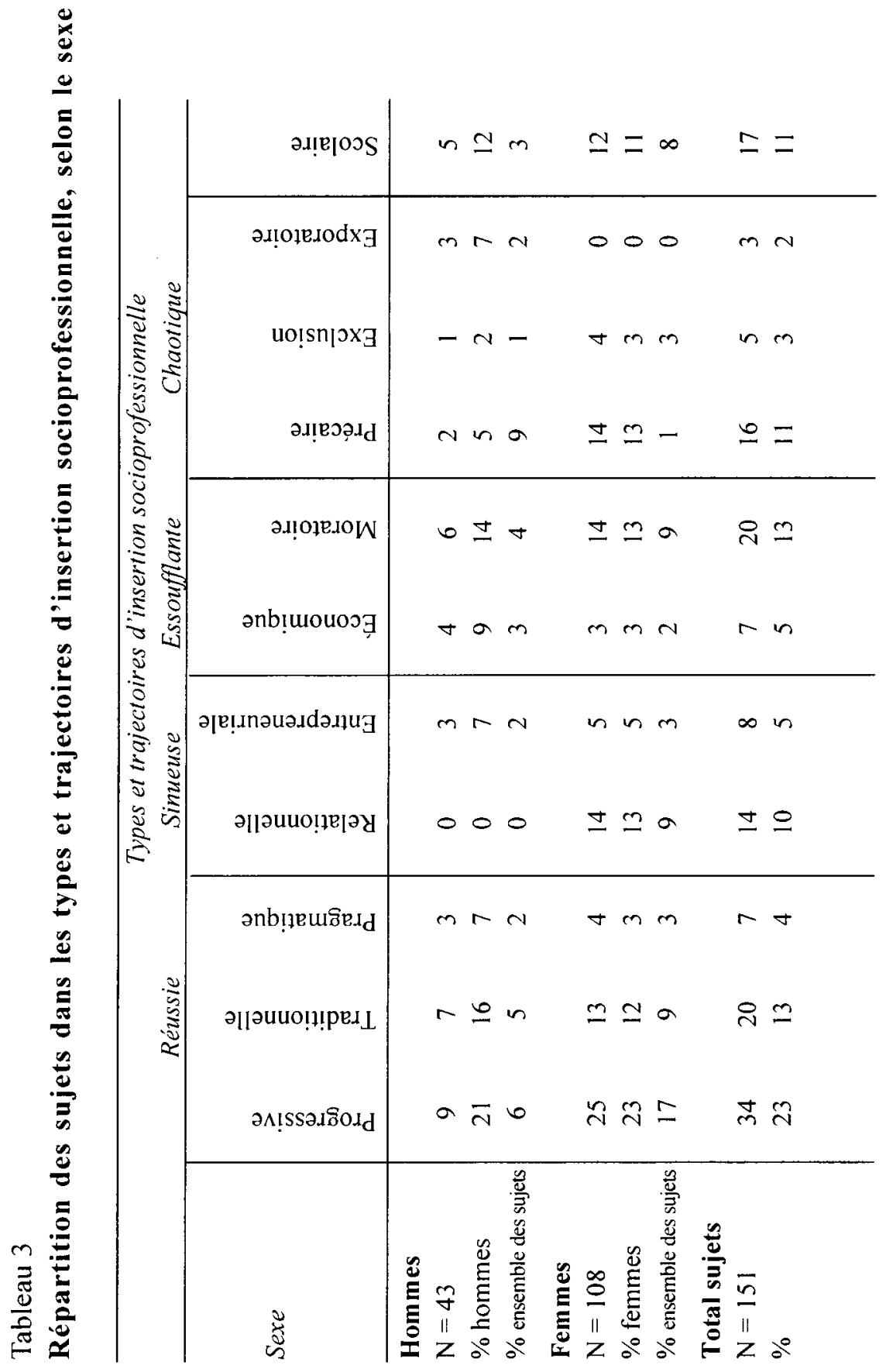

The Canadian Journal of Higher Education Volume XXXII, No. 3, 2002 
répartition des hommes et des femmes est comparable dans les trajectoires progressive, traditionnelle et moratoire.

\section{DISCUSSION}

Entre la situation d'emploi typique et l'exclusion se dessinent de multiples voies d'insertion socioprofessionnelle: onze trajectoires différentes, plus ou moins choisies, plus ou moins bien vécues par les individus qui les expérimentent, mais qui confèrent une autre dimension à l'espace d'insertion entre la fin des études et l'accès à un emploi satisfaisant.

La réalité des nouveaux diplômés présentée dans cette. recherche met en lumière à quel point la représentation d'un parcours d'insertion socioprofessionnelle linéaire et prévisible, longtemps associée aux diplômés, est aujourd'hui obsolète ou du moins réductrice. En effet, parmi l'échantillon étudié dans le cadre de cette étude, peu de sortants du système éducatif ont accédé immédiatement à un emploi pour y demeurer et gravir progressivement les échelons de l'entreprise. En fait, les sujets les plus choyés, qui sont regroupés dans les trois trajectoires du type d'insertion réussie (40\%), occupent, après trois ans sur le marché de l'emploi, une position stable et satisfaisante, mais ils y sont généralement parvenus après deux ou trois emplois différents. Cependant, selon Bordigoni et Mansuy (1997), cette mobilité est normale et ne doit pas être systématiquement assimilée à un épisode de précarité:

S'il est courant d'admettre que dans la carrière d'un cadre, un changement d'emploi s'accompagne d'une amélioration de la rémunération, cela constitue aussi une réalité pour des jeunes ouvriers ou employés en début de carrière. Il ne faut pas interpréter la mobilité chez ces jeunes que comme fatum baptisé précarité. (p. 116)

La mobilité des sujets du type d'insertion réussie présente souvent cette stratégie de progression vers un meilleur emploi ou un salaire plus avantageux.

Pour les autres répondants $(60 \%)$, l'insertion sur le marché du travail s'est révélée un parcours semé d'embûches, où les déceptions sont d'autant plus cuisantes que les efforts et les sacrifices consentis pour y 
parvenir ont été importants. Cependant, certains s'accommodent plutôt bien de leur état, comme les femmes de la trajectoire relationnelle $(9 \%)$ qui ont choisi de concentrer leurs énergies sur leur vie familiale et sociale plutôt que sur leur vie professionnelle. C'est le cas également des sujets de la trajectoire économique $(5 \%)$ qui, pressés de subvenir à leurs besoins personnels ou à ceux de leur famille, acceptent un emploi peu valorisant et hors de leur domaine de formation. Ils attribuent alors à leur travail une fonction instrumentale, leur permettant de maintenir un équilibre dans les autres sphères de leur vie.

D'autres encore ont choisi, d'une certaine manière, la situation d'instabilité dans laquelle ils se trouvent. On retrouve ici les entrepreneurs (5\%), qui ont voulu démarrer une entreprise ou se sont constitués travailleurs autonomes pour être maîtres de leur situation d'emploi. Cette trajectoire se rapproche de celle identifiée par Gauthier (1994) et nommé l'instabilité créatrice. Les travailleurs qui s'y trouvent «...savent contourner stratégiquement les difficultés et les barrières qui constituent des obstacles pour certains et l'exclusion pour les plus fragiles» (Gauthier, 1994, p. 160). Ils admettent cependant qu'une période plus difficile, au plan financier surtout, doit être surmontée avant de pouvoir aspirer à davantage de stabilité. Les jeunes hommes de la trajectoire exploratoire $(2 \%)$ ont également, dans une certaine mesure, choisi leur situation puisqu'ils ont opté pour une insertion du marché de l'emploi avant la fin de leurs études collégiales. En quête d'identité professionnelle, ils évoluent d'un boulot à l'autre et y retirent une certaine satisfaction, même si cette trajectoire les maintient dans un contexte de précarité professionnelle et personnelle.

En revanche, les sujets de trois trajectoires subissent sans conteste plus péniblement les aléas d'une insertion essoufflante, dans le cas de la trajectoire moratoire, ou d'une insertion chaotique, dans le cas des trajectoires précaire et d'exclusion. Les uns, ceux de la trajectoire moratoire (13\%) ont abandonné leur projet professionnel, découragés par un marché du travail perçu comme hermétique et se sont rabattus sur un emploi stable, mais mal rémunéré, éloigné de leur formation et peu gratifiant. Gauthier (1994) a également reconnu un type d'insertion de jeunes qui en viennent à se trouver satisfaits d'être en emploi, peu 
importe les conditions, pourvu qu'ils travaillent. Cependant, au sujet de ce type désigné sous l'appellation la stabilité dans la précarité, elle écrit:

Peut-on aussi qualifier ces jeunes de «gagnants»? Ils sont gagnants par comparaison avec ceux qui se trouvent en chômage de longue durée ou constamment en recherche d'emploi à cause de la durée limitée des emplois qu'ils obtiennent. Ces gagnants peuvent multiplier les emplois au cours d'une année, mais ils ne sont généralement pas longtemps en chômage. Ils sont des perdants en ce qu'ils écopent des emplois les moins intéressants sur un marché détérioré. (p. 163)

Les autres qui vivent plus difficilement leur situation, ceux des trajectoires précaire (11\%) et d'exclusion (3\%), maintiennent leur projet professionnel, mais sans parvenir à l'atteindre, par défaut d'employabilité ou parce que les perspectives d'insertion professionnelle sont particulièrement obstruées dans leur domaine de formation. Les sujets de ces deux trajectoires vivent dans des conditions d'instabilité et de dépendance économique importantes, nuisant à leur qualité de vie et bloquant la mise en œuvre de projets dans toutes les sphères de leur vie.

La difficulté d'insertion de certains individus deviendrait davantage problématique selon le temps passé dans des emplois peu qualifiants ou selon le moment où survient une période difficile. Ainsi, Vernières (1997) souligne que «Moins le temps passé dans des positions peu qualifiantes est grand, plus semble donc efficient le processus d'insertion» (p. 18). Ce qui semble être le cas inverse des sujets de la trajectoire moratoire qui expérimentent une succession de petits boulots, d'entrées et de sorties du marché du travail jusqu'à ce qu'ils acceptent un emploi stable, hors de leur domaine et en-deçà de leurs qualifications. Ils s'accrochent alors à cette stabilité déstabilisante pour éviter de reprendre la ronde de la précarité. Par ailleurs, Vernières (1997) convient des difficultés particulières liées au moment où surviennent les périodes difficiles:

En cas de cheminement avec de nombreuses successions d'états, la sommation du temps passé dans chacun d'eux paraît donc comme un bon indicateur de la qualité du processus d'insertion. Mais il convient également d'analyser 
l'ordre et le rythme de ces passages. Être au chômage, en stage d'initiation ou en emploi précaire non qualifiant en fin de période est, à durée égale, plus grave qu'en début de période. Un passage fréquent, tout au long de la période, d'une position à une autre, traduisant un cheminement très instable et complexe, est signe, a priori, d'un processus difficile. (p. 18)

Ce propos confirme la position difficile qu'occupent les gens des trajectoires précaire, exploratoire et d'exclusion, trois ans après leur sortie du système éducatif. Si la trajectoire suivie ressemble à plusieurs égards à celle des sujets qui s'inscrivent dans la trajectoire moratoire, il n'en demeure pas moins que, différemment des autres, ils n'occupent pas d'emplois stables et que leur survie économique est menacée. Les sujets qui retournent à l'école ont un parcours qui peut se comparer à celui de la trajectoire précaire mais ils ont choisi cette voie pour se sortir du cercle vicieux dans lequel ils se trouvaient. Cependant, il ne faut pas banaliser le fait que cette décision soit fort coûteuse pour plusieurs et qu'elle survienne à la suite d'une incapacité de trouver sur le marché du travail des possibilités de réalisation de leur choix professionnel initial. En somme, il s'agit d'une décision personnelle, certes, mais motivée par un échec dans la quête d'un espace cohérent avec l'investissement scolaire et réalisable uniquement pour ceux et celles qui bénéficient d'un soutien financier.

En ce qui a trait à la distribution des individus selon le sexe, le cas de la trajectoire relationnelle, occupée uniquement par des femmes, en grande majorité diplômée de l'université de surcroît, est particulièrement frappant. En effet, il est étonnant de constater que ce sont uniquement des femmes qui ont décidé de privilégier leur vie hors travail plutôt que leur vie professionnelle, et ce, en début de carrière. Trois raisons principales ont été évoquées en entrevue pour expliquer cette décision. D'abord, en plus des déceptions ressenties à l'égard des emplois offerts, certaines femmes ont mentionné avoir préféré accepter des emplois moins intéressants, mais dans leur région immédiate, plutôt que de s'éloigner et de mettre en péril leur relation de couple dans laquelle elles se sentaient déjà très engagées. D'autres ont jugé préférable de devancer 
de quelques années leur projet de maternité et de reporter à plus tard leur projet d'insertion compte tenu du peu d'intérêts des emplois disponibles. Enfin, quelques-unes ont rapporté avoir refusé l'isolement social que peut entraîner le déménagement dans une région éloignée et avoir opté pour un emploi instable et parfois sans lien avec leur formation, plutôt qu'un emploi stable mais loin de leur famille, de leurs amis ou de leur amoureux. Bien qu'elles auraient souhaité un meilleur emploi, celles-là, tout comme les autres femmes appartenant à la trajectoires relationnelle, ont mentionné que les compromis à faire pour occuper un emploi stable et en lien avec leur formation, constituait une menace trop sérieuse à la qualité de leur vie personnelle et sociale.

Jusqu'à un certain point, la trajectoire économique peut être vue comme le corollaire masculin de la trajectoire relationnelle. Si elle diffère sur certains points majeurs dont la stabilité de l'emploi, il n'en demeure pas moins que plusieurs de ces sujets ont choisi d'accepter un emploi hors de leur domaine d'études pour garantir la qualité de leur vie personnelle. La satisfaction de pouvoir " rapporter de l'argent à la maison» et de subvenir aux besoins de leurs proches est à ce moment de leur processus d'insertion, plus importante que la réalisation immédiate de leurs projets professionnels. Comme les femmes de la trajectoire relationnelle, les conditions structurelles du marché du travail mais surtout des choix de vie personnelle sont venus interférer dans le processus d'insertion socioprofessionnelle et en ont différé la trajectoire. Cependant, les motifs invoqués pour justifier de tels choix et le contexte dans lequel ils ont été faits, sont fort différents de ceux de la trajectoire relationnelle: les sujets de la trajectoire économique ont tous mentionné avoir fait face à des événements personnels inattendus qui ont entraîné des obligations financières imprévues. L'obtention d'un emploi stable et bien rémunéré est alors devenue un impératif dont dépendait la qualité de vie personnelle. Si, dans les deux cas, c'est cette qualité de vie personnelle qui a été privilégiée, on constate que dans le premier cas (trajectoire relationnelle), la vie au travail a été reléguée délibérément au second plan tandis que dans le second cas (trajectoire économique), la vie au travail est demeurée prioritaire mais elle a pris, compte tenu des événements de vie personnelle, une valeur nettement plus instrumentale, voire utilitaire. 
D'autre part, il est intéressant de considérer plus précisément que les individus issus du secondaire se trouvent satisfaits de leur insertion dans une proportion significative: $46 \%$ d'entre eux se retrouvent dans le type d'insertion réussie, contre $27 \%$ des collégiens et $52 \%$ des universitaires. Ceci permet de constater l'efficacité des programmes d'études professionnelles au secondaire, qui font l'objet depuis quelques années d'une importante remise à niveau, surtout si on compare le taux d'insertion réussie du secondaire et du collégial technique. Plus précisément, les diplômés du cégep sont les plus nombreux (21\%) à se trouver dans le type d'insertion chaotique, où l'on retrouve les trajectoires les plus difficiles. Ils sont aussi beaucoup plus nombreux à retourner sur les bancs d'école (21\%) peu de temps après l'obtention de leur diplôme.

En somme, cette typologie et la distribution des sujets en fonction de leur niveau de scolarité et de leur sexe permettent de mieux comprendre le processus d'insertion socioprofessionnelle des nouveaux diplômés. La perspective qualitative retenue pour cette étude a en outre comblé une certaine lacune dans l'appréhension de cet objet d'étude en dégageant le sens de l'expérience vécue par les individus eux-mêmes. En effet, certaines constantes ressortent clairement de cette expérience. D'abord, pour rendre compte de leur trajectoire d'insertion socioprofessionnelle, les sujets font appel à des situations de leur vie tant personnelle que professionnelle qui sont souvent ni planifiées ni anticipées. L'enchevêtrement entre les sphères de vie privée et professionnelle est complexe et souvent décisif dans la trajectoire. Ensuite, le réseau de références professionnelles est un des facteurs le plus invoqué par les sujets pour expliquer la réussite de leur insertion de même que l'accès à un soutien financier qui offre la latitude nécessaire pour mener à terme un cheminement d'insertion souvent long et périlleux. Troisièmement, plusieurs jeunes diplômés sont obligés d'ajuster ou de différer leurs attentes, objectifs et projets professionnels pour demeurer disponibles aux possibilités qui se présentent ou simplement pour s'affranchir de leur situation de précarité. Enfin, des choix déchirants ponctuent l'expérience d'insertion d'un bon nombre de jeunes. Pour se maintenir en emploi ou pour acquérir une expérience de travail valable, plusieurs sont appelés à faire des concessions sur leur investissement en dehors du travail de même 
qu'à s'éloigner de leur réseau de soutien social (famille, amis et conjoint). Certains acceptent de faire ces concessions. D'autre estiment que le prix est trop lourd à payer et refusent de négocier la qualité de leur vie personnelle et sociale.

Finalement, certaines questions relatives à l'insertion de l'ensemble des jeunes, diplômés et non-diplômés, émergent de cette étude. En effet, si parmi notre échantillon de diplômés, réputés s'insérer plus facilement sur le marché de l'emploi, seul un petit nombre a « réussi » leur insertion trois ans après leur sortie de l'école, qu'en est-il des individus non diplômés? Qu'en est-il également de ceux qui suivent une formation où le taux de placement est faible, ou de ceux qui sont mal préparés à entreprendre des démarches de recherche d'emploi dans le contexte de compétition que l'on connaît? Quoi qu'il en soit, cette étude et les résultats avancés démontrent que l'insertion socioprofessionnelle des diplômés constitue une réalité aux multiple visages, qu'il serait risqué de considérer de façon homogène.

\section{Les Notes}

1 Il est à noter que sept sujets, soit $5 \%$ de l'échantillon, n'ont pas obtenu leur diplôme. Ils ont tout de même été intégrés à l'analyse des données.

2 À la $3^{\mathrm{c}}$ année de la recherche, 54 sujets on refusé de continuer de participer à la recherche pour diverses raisons: i.e., manque d'intérêt, manque de temps, déménagement dans une région éloignée ou à l'extérieur du pays, etc.

${ }^{3}$ Contrat à durée indéterminée et contrat à durée déterminée.

\section{Bibliographie}

Aubry, F. (1998). Le plein emploi: Mythe ou réalité?. Dans D.G. Tremblay (Ed.), Objectif plein emploi (pp. 85-101). Ste-Foy, QC: Presses de l'Université du Québec.

Baby, A. (2000). Le travail «atypique» vu depuis la face cachée de la lune. Dans G. Fournier, \& B. Bourassa (Eds.), Les 18-30 ans et le marché du travail. Quand la marge devient la norme... (pp. 219-241). Québec, QC: Les Presses de l'Université Laval. 
Bardin, L. (1991). L'analyse de contenu (6e éd.). Paris: Presses Universitaire de France.

Béret, P. (1996). Projet professionnel et insertion des jeunes. Dans B. Francq, \& C. Maroy (Eds.), Formation et socialisation au travail (pp. 167-181). Paris: De Boeck et Larcier.

Bordigoni, M., \& Mansuy, M. (1997). Les parcours professionnels des lycéens et apprentis débutants. Économie et statistique. 304-305, 109-120.

Bovin, B. (1995). Education, employment and unemployment. Dans OCDE (Eds.), Formation et emploi, indicateurs des systèmes d'enseignement (pp. 70-83). Paris: OCDE.

Canals, V. (1998). Les formes précaires d'emploi dans les premiers temps de la vie active. Revue de l'économie méridionale, 46, 107-129.

Castel, R. (1995). Les pièges de l'exclusion. Lien social et Politiques, 34, 13-21.

Chalifour, S. (1997). La question sociale et la réduction du temps de travail. Travailler autrement. Vivre mieux?. Possibles, 2l(2), 47-64.

Conseil permanent de la jeunesse. (2001). Emploi typique et précarité chez les jeunes. Une main-d'auvre à bas prix, compétente et jetable! Québec, QC: Gouvernement du Québec.

Dagenais, L.-F. (1998). La question des jeunes et la stratégie d'emploi jeunesse. Éléments de réflexion. Cahiers de recherche sociologique, 31, 53-79.

Fortier, Y. (2000). La fin du travail cinq ans plus tard: la proportion de la population qui occupe un emploi n'a jamais été aussi élevée. Québec, QC: Centre d'étude sur l'emploi et la technologie, Direction de la planification et de l'information sur le marché du travail, Emploi-Québec.

Fournier, G., Boivin, M.-D., \& Viel, D. (1999). L'individu au cœur des pratiques éducatives d'aide à l'insertion socioprofessionnelle. Cahiers de la recherche en éducation, 5(2),19-44.

Fournier, G., \& Bourassa, B. (2000). Le travail des 18-30 ans: Vers une nouvelle norme. Dans G. Fournier, \& B. Bourassa (Eds.), Les $18-30$ ans et le marché du travail. Quand la marge devient la norme... (pp. 3-27). Québec, QC: Les Presses de l'Université Laval.

Fournier, G., Jeanrie, C., \& Croteau, L. (1999). Qualité de l'insertion socioprofessionnelle de nouveaux travailleurs diplômés: Examen d'un schéma exploratoire. Revue Canadienne de l'Enseignement Supérieur, 29(1), 143-177. 
Fournier, G., Monette, M., Pelletier, R., \& Tardif, P. (2000). Les diplômée-s et l'insertion socioprofessionnelle: Résignation déguisée ou adaptation saine à un marché du travail insensé. Dans G. Fournier, \& M. Monette (Eds.), L'insertion socioprofessionnelle: Un jeu de stratégie ou un jeu de hasard? (pp. 1-32). Québec, QC: Les Presses de l'Université Laval.

Fusulier, B. (1996). L'insertion juvénile: Une mise à l'épreuve de soi. Recherches sociologiques, 3, 27-36.

Gauthier, M. (1994). Une société sans les jeunes? Québec, QC: Institut québécois de recherches sur la Culture.

Gorz, A. (1997). Misères du présent, richesse du possible. Paris: Éditions Galilée.

Kahn, S. (1997). L'orientation, l'affaire de chacun ou l'affaire de tous? Dans Collectif (Eds.), L'orientation face aux mutations du travail (pp. 15-24). Paris: Syros.

Laflamme, C. (2000). La poursuite des études: une question de pouvoir du diplôme et de marginalisation. Dans G. Fournier, \& B. Bourassa (Eds.), Les 18-30 ans et le marché du travail. Quand la marge devient la norme... (pp. 119-138). Québec, QC: Presses de l'Université Laval.

L'Écuyer, R. (1990). Méthodologie de l'analyse développementale de contenu: Méthode GPS et concept de soi. Sillery, QC: Presses de l'Université du Québec.

Lollivier, S. (2000). Récurrence du chômage dans l'insertion des jeunes: Des trajectoires hétérogènes. Économie et statistique, 334, 49-63.

Nicole-Drancourt, C. (1992). L'insertion professionnelle des jeunes garçons et filles: une étude localisée. Dans L. Coutrot, \& C. Dubar (Éds.), Parcours professionnels et mobilités sociales (pp. 285-298). Paris: La documentation française.

Nicole-Drancourt, C. (1997). Accéder à un emploi. Projet, 25l, 20-30.

Nicole-Drancourt, C., \& Roulleau-Berger, L. (1995). L'insertion des jeunes en France. France: Presses universitaires de France.

Nicole-Drancourt, C., \& Roulleau-Berger, L. (2000). Les jeunes et le travail, 1950-2000. Paris: Presses universitaires de France.

Onimus, J. (1997). Quand le travail disparaitt. Paris: Desclée De Brouwer

Pair, C. (1998). L'orientation dans un monde incertain. L'enseignement technique, 177, 3-10.

Paugam, S. (2000). Le salarié de la précarité. Paris: Presses universitaires de France. 
Perret, B. (1995). L'avenir du travail. Les démocraties face au chômage. Paris: Seuil.

Pottier, F. (1992). Formes et logiques de mobilité des jeunes à travers l'observatoire des entrées dans la vie active (EVA). Dans L. Coutrot, \& C. Dubar (Éds.), Parcours professionnels et mobilités sociales (pp. 259-284). Paris: La documentation française.

Rebière, C. (1998). L'insertion professionnelle des lycéens sept mois après leur sortie du système éducatif: Situation au ler février 1998. Note d'information. 6(5), 1-6.

Rose, J. (1994). L'organisation des transitions professionnelles entre socialisation, mobilisation et recomposition des rapports de travail et d'emploi. Sociologie du travail, 38, 1, 63-79.

Rose, J. (1998). Les jeunes face à l'emploi. Paris: Desclée de Brouwer

Rose, J. (2000). Les jeunes et l'emploi:questions conceptuelles et méthodologiques. Dans G. Fournier, \& B. Bourassa (Eds.), Les 18-30 ans et le marché du travail. Quand la marge devient la norme... (pp. 83-116). Québec, QC: Les Presses de l'Université Laval.

Tremblay, D.G. (1994). Chômage, flexibilité et précarité d'emploi: Aspects sociaux. Dans F. Dumont, S. Langlois, \& Y. Martin (Eds.), Traité des problèmes sociaux (pp. 623-652). Québec, QC: Institut québécois de recherche sur la culture.

Trottier, C. (2000). Le rapport au travail et l'accès à un emploi stable, à temps plein, lié à la formation : vers l'émergence de nouvelles normes? Dans G. Fournier, \& B. Bourassa (Eds.), Les 18-30 ans et le marché du travail. Quand la marge devient la norme... (pp.35-58). Québec, QC: Les Presses de l'Université Laval.

Trottier, C., Perron, M., \& Diambomba, M. (1995).Les cheminements scolaires et d'insertion professionnelle des étudiants de l'Université. Québec, QC: Les Presses de l'Université Laval.

Vernières, M. (1993). L'insertion professionnelle. Formation Emploi. Enjeu économique et social. Paris: Éditions Cujas.

Vernières, M. (1997). L'insertion professionnelle, analyses et débats. Paris: Économica.

Vincens, J. (1998). L'insertion professionnelle des jeunes. A la recherche d'une définition conventionnelle. Formation emploi, 60, 21-36. 